\title{
Nonequilibrium kinetics of a disordered Luttinger liquid
}

\author{
D. A. Bagrets ${ }^{1}$, I. V. Gornyi ${ }^{1,2}$, and D. G. Polyakov ${ }^{1}$ \\ ${ }^{1}$ Institut für Nanotechnologie, Forschungszentrum Karlsruhe, 76021 Karlsruhe, Germany \\ ${ }^{2}$ A.F.Ioffe Physico-Technical Institute, 194021 St. Petersburg, Russia
}

(Dated: October 22, 2018)

\begin{abstract}
We develop a kinetic theory for strongly correlated disordered one-dimensional electron systems out of equilibrium, within the Luttinger liquid model. In the absence of inhomogeneities, the model exhibits no relaxation to equilibrium. We derive kinetic equations for electron and plasmon distribution functions in the presence of impurities and calculate the equilibration rate $\gamma_{E}$. Remarkably, for not too low temperature and bias voltage, $\gamma_{E}$ is given by the elastic backscattering rate, independent of the strength of electron-electron interaction, temperature, and bias.
\end{abstract}

PACS numbers: 71.10.Pm 05.30.Fk 05.60.Gg 73.21.Hb

Introduction.--Interacting electrons in one dimension (1D) [1] have become a focus of interest in nanophysics from both the fundamental and applied perspectives. Recent technological advances have made it possible to systematically study the transport properties of a variety of ultranarrow wires; in particular, carbon nanotubes [2] and semiconductor nanowires 3]. From the fundamental point of view, much of the fascination with physics of $1 \mathrm{D}$ systems is driven by the fact that electron-electron (e-e) interactions in $1 \mathrm{D}$ geometry are qualitatively significant, transforming the electron gas into a Luttinger liquid (LL) [1]. This strongly correlated state of matter is commonly described in terms of bosonic elementary excitations.

A conceptually nontrivial aspect of the non-Fermi liquid nature of a LL concerns its behavior at nonequilibrium, e.g., when a finite bias voltage is applied to the wire (for a recent experiment see Ref. [4]). A homogeneous LL is completely integrable and as such does not exhibit any relaxation to equilibrium: an excited state will never decay to the state characterized by temperature T. Quite remarkably, a finite quantum lifetime of fermionic excitations due to e-e interactions in a homogeneous LL does not translate into any inelastic e-e scattering [5]: the allowed energy transfer is exactly zero. This is in stark contrast to electron liquids in higher dimensions, where the characteristic energy transfer is $T$. Relaxation to equilibrium due to e-e collisions in a LL is thus only possible if momentum conservation is broken by inhomogeneities. Of central importance is therefore the question - essentially unanswered - of how the equilibration in a LL occurs in the presence of a random backscattering potential. This is the subject of this work.

So far, advances in dealing with a LL off equilibrium have been focused on a "mechanical" approach, i.e., on solving the equations of motion "as exactly as possible". Efforts, based on the bosonization approach, have been centered around the nonlinear conductance of a LL containing a single compact scatterer [6]. However, the line of research relying on the exact integrability cannot possibly be much extended beyond the single-scatterer case (for two tunneling barriers in a LL, a nonequilibrium dis- tribution of plasmons was studied by means of the master equation, see Ref. [7]). Also, importantly, the conventional bosonization [1] is designed for equilibrium boundary conditions. An alternative is to preserve dynamics of both bosonic and fermionic degrees of freedom [8, 9], as it is done in the functional bosonization approach (see Ref. [10] for a review).

Our purpose here is to develop a kinetic approach to nonequilibrium phenomena in a disordered LL, by formulating kinetic equations for distribution functions. Within this approach, one has to introduce the distribution functions of not only bosonic but also fermionic excitations, similarly to higher dimensions [11, 12]. Our main result is a set of kinetic equations which describe (i) inelastic e-e scattering, mediated by virtual plasmons, and (ii) creation/annihilation of real plasmons; both processes being only triggered by scattering off disorder. We calculate a key quantity in nonequilibrium problems: the energy relaxation rate $\gamma_{E}$. In a remarkable departure from Fermi liquids, $\gamma_{E}$ in a LL at not too low $T$ turns out to be given by the elastic scattering rate $\gamma$.

Effective action.-We study interacting electrons in a single-channel disordered quantum wire within the LL model [1]: the electron dispersion relation is linear (with the velocity $v_{F}$ ) and interactions yield only forward e-e scattering (characterized by the dimensionless constant $\alpha=V_{\mathrm{f}} / 2 \pi v_{F}$, where $V_{\mathrm{f}}$ is the zero-momentum Fourier component of the interaction potential). We consider both spinless $(\eta=1)$ and spinful $(\eta=2)$ models. Our approach is based on the "quasiclassical" real-time electron Green's function at coinciding spatial points $\hat{g}\left(x, t_{1}, t_{2}\right)$ [13], widely used in the nonequilibrium theory of mesoscopic transport. The "hat" means that $\hat{g}$ is a matrix in the Keldysh, chirality ( $\mu= \pm$ for right/left-moving electrons) and (possibly) spin $(s=\uparrow, \downarrow)$ spaces. In what follows we use the Pauli matrices $\tau, \sigma$, and $s$ that act in the chirality, Keldysh, and spin spaces, respectively.

The quasiclassical Green's function satisfies the condition $\hat{g} \circ \hat{g}=\delta\left(t_{1}-t_{2}\right)$, where the "dot" denotes the convolution in the full (chirality $\times \operatorname{spin} \times$ Keldysh $\times$ time) space. This constraint enables us to describe the wire 
by the action that reproduces the averaged over disorder equation of motion for $\hat{g}$ as its saddle point. The way to derive such an action is similar to that in the ballistic sigma-model in higher dimensions [14]. To account for the Coulomb interaction, one introduces the Hubbard-Stratonovich field $\hat{\phi}(x, t)=\phi_{1}+\sigma_{x} \phi_{2}$ on the Keldysh contour [15], where the "classical" and "quantum" fields $\phi_{1,2}$ are diagonal matricies in the chirality and spin spaces. Then the action takes the form [8]

$$
\begin{aligned}
S\{\hat{g}, \hat{\phi}\} & =\frac{1}{2} \operatorname{Tr} \int d x\left[\frac{1}{v_{F}^{*}}\left(-i \partial_{t}+\hat{\phi}\right) \tau_{z} \hat{g}-\hat{g}_{0} \mathcal{T}^{-1} i \partial_{x} \mathcal{T}\right. \\
& \left.-\frac{i \gamma_{0}}{16 v_{F}^{*}} \tau_{+} \hat{g} \tau_{-} \hat{g}+\hat{\phi}\left(\frac{\sigma_{x}}{2 \pi v_{F}^{*}}+\hat{V}_{0}^{-1}\right) \hat{\phi}\right] .
\end{aligned}
$$

Here $\gamma_{0}$ is the bare elastic rate of backscattering off a random static potential, $\hat{g}_{0}=\operatorname{diag}\left(g_{0}^{+},-g_{0}^{-}\right)$corresponds to the saddle point of the action of the noninteracting problem, and $\hat{g}=\mathcal{T} \hat{g}_{0} \mathcal{T}^{-1}=\operatorname{diag}\left(g^{+},-g^{-}\right)$. The unitary transformation $\mathcal{T}$ (diagonal in the chirality $\times$ spin space) parametrizes fluctuations around $\hat{g}_{0}$ due to fluctuations of $\hat{\phi}(x, t)$. The interaction of fermions of the same chirality and spin is included in the shift of the Fermi velocity $v_{F}^{*}=v_{F}+V_{\mathrm{f}} / 2 \pi[5]$. The last term in Eq. (11) with $\hat{V}_{0}^{-1}=V_{\mathrm{f}}^{-1} \sigma_{x} \tau_{x}\left[1+2(\eta-1) s_{x}\right]$ accounts for true interactions. A similar (replicated imaginary-time) action for a disordered LL at equilibrium was proposed in Ref. [16].

Quantum kinetic equations.- Starting from the effective Keldysh action (11), we use the standard procedure [13, 17] to derive the kinetic equations. We proceed at one-loop order with respect to the effective interaction, which is equivalent to the "dirty random-phase approximation" (dRPA) [5]. The one-loop derivation is controlled by the parameters $\gamma / \max \{T, e U\} \ll 1$ and $\alpha \ll 1$, which is assumed in the rest of the paper $(U$ is the bias voltage). We also disregard the localization effects [ [5, 18]. For a wire of length $L \gtrsim v_{F} / \gamma$, this limits the applicability of what follows to $\max \{T, e U\} \gg T_{1}=\gamma / \alpha^{3-\eta}$.

Within the dRPA, we expand the action (11) in fast (on a scale of the relaxation time for the kinetic equation) quadratic fluctuations of $\hat{\phi}$ around the "slow" semiclassical saddle point $g_{\text {slow }}^{\mu}=\left(\sigma_{z}+\sigma_{+}\right) \delta\left(t_{1}-\right.$ $\left.t_{2}\right)-2 \sigma_{+} f^{\mu}\left(x, t_{1}, t_{2}\right)$. The electron distribution function $f_{\epsilon}^{\mu}(x, t)$ at given energy $\epsilon$ is defined via the Wignertransform of $f^{\mu}\left(x, t_{1}, t_{2}\right)$ and is yet to be found from the kinetic equations. In this way we obtain the Gaussian action with the propagator $\hat{V}=\left(\hat{V}_{0}^{-1}-\hat{\Pi}\right)^{-1}$. Here $\hat{\Pi}=\sigma_{x}\left(\partial_{t} \hat{D} \sigma_{x}-1\right) / 2 \pi v_{F}^{*}$ is the polarization operator, with $\hat{D}$ being the electron-hole (e-h) propagator damped by disorder. The retarded part of $\hat{D}$ is given by $D_{R}^{-1}=-i\left(\omega-\tau_{z} v_{F}^{*} q\right)+\gamma\left(1-\tau_{x}\right) / 2$, while the kinetic part $D_{K}$ is expressed (see below) in terms of $f_{\epsilon}^{\mu}$ via

$$
N_{\omega}^{\mu \nu}=\frac{1}{2 \omega} \int d \epsilon\left[f_{\epsilon}^{\mu}\left(1-f_{\epsilon-\omega}^{\nu}\right)+(\mu \leftrightarrow \nu)\right] .
$$

To derive the kinetic equation for electrons, we average

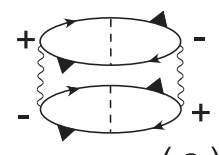

(a)

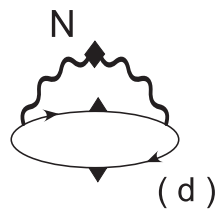

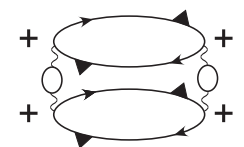

(b)

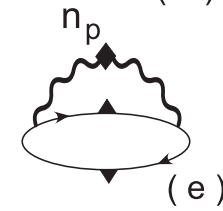

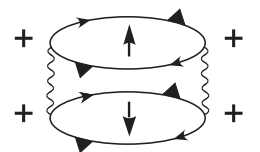

(c)

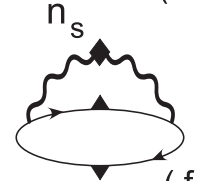

( f)
FIG. 1: Scattering processes corresponding to the collision kernels $\mathcal{K}(\omega)$ and $\mathcal{L}(\omega)$. Thin wavy lines: bare interaction $V_{\mathrm{f}}$. Solid wavy lines: dRPA interaction propagator $V_{>}$. The triangles $(\mathbf{\Lambda})$ and rhombi $(\checkmark)$ mean the Keldysh part of the electron and boson Green's functions, respectively.

out the fast fluctuations in the equation of motion for $\hat{g}\left(x, t_{1}, t_{2}\right)$ with the dRPA action, which yields [19]

$$
\left(\partial_{t}+\mu v_{F}^{*} \partial_{x}\right) f_{\epsilon}^{\mu}=-\frac{\gamma+\gamma_{\mathrm{inel}}}{2}\left(f_{\epsilon}^{\mu}-f_{\epsilon}^{-\mu}\right)+\mathrm{St}_{e-b}^{\mu}(\epsilon) .
$$

The collision integral $\mathrm{St}_{e-b}^{\mu}$ describes inelastic electron scattering due to interaction with the bosonic bath,

$$
\mathrm{St}_{e-b}^{\mu}=\sum_{\nu} \int d \omega I^{\mu \nu}(\omega)\left[f_{\epsilon+\omega}^{\nu}\left(1-f_{\epsilon}^{\mu}\right)-f_{\epsilon}^{\mu}\left(1-f_{\epsilon-\omega}^{\nu}\right)\right],
$$

while $\gamma_{\text {inel }}$ accounts for the additional [19] renormalization of the static disorder due to the inelastic scattering,

$$
\frac{\gamma_{\text {inel }}}{2}=-\int d \omega I^{+-}(\omega)\left(1-f_{\epsilon-\omega}+f_{\epsilon+\omega}\right) .
$$

Here $f_{\epsilon}=\left(f_{\epsilon}^{+}+f_{\epsilon}^{-}\right) / 2$, and $I^{\mu \nu}(\omega)$ is the rate of emission of energy per unit interval of $\omega$, accompanied by scattering $\mu \rightarrow \nu$, which is given by

$$
I^{\mu \nu}(\omega)=\frac{i}{\pi} \int \frac{d q}{2 \pi} V_{>, \|}^{\nu \mu}(\omega, q) \operatorname{Re} D_{R}^{\mu \nu}(\omega, q) .
$$

In the integrand (6), there are four poles, $q \simeq \pm \omega(1 \pm$ $i \gamma / 2 \omega) / v_{F}^{*}$, inherited from $\operatorname{Re} D_{R}$, which are only slightly damped by disorder in the limit $\omega \gg \gamma$. They correspond to e-h pair excitations described by the renormalized Fermi velocity $v_{F}^{*}$. Four more poles $q \simeq \pm \omega(1 \pm i \gamma / 2 \omega) / u$, associated with the "greater" part of the effective interaction with parallel spins $V_{>, \|}$, correspond to the collective plasmon mode of the clean LL, moving with velocity $u=v_{F}(1+\eta \alpha)^{1 / 2}$. In the spinful model, $\hat{V}$ contains an extra mode - spinon-propagating with velocity $v_{F}$ [18]. Importantly, the e-h and collective excitations at $\omega \gg T_{1}$ are well resolved from each other and should be treated separately, while in the opposite limit, $\omega \ll T_{1}$, the disorder-induced quantum uncertainty makes them indistinguishable. We now proceed by relating $V_{>, \|}^{\nu \mu}$ to the distribution functions.

Small energy transfer, $\omega \ll T_{1}$. - In this limit, the Keldysh, retarded, and advanced parts of interaction satisfy $V_{K} \simeq V_{R} \Pi_{K} V_{A}$. Then the general result for the 
emission rate can be represented in the form $I^{\mu \nu}(\omega)=$ $\sum_{\alpha \beta} \omega \mathcal{K}_{\alpha \beta}^{\mu \nu}(\omega) N_{\omega}^{\alpha \beta}$, where the collision kernel $\mathcal{K}_{\alpha \beta}^{\mu \nu}(\omega)$ describes inelastic spin-conserving electron scattering $\mu \rightarrow \nu$ with energy transfer $\omega$ to the electron and hole having the chirality $\alpha$ and $\beta$. For the total collision kernel $\mathcal{K}=\frac{1}{2} \sum_{\mu \nu} \mathcal{K}_{\mu \nu}^{\mu \nu}$ we obtain

$$
\mathcal{K}(\omega)=\int \frac{d q}{2 \pi^{2} \omega} \sum_{\mu \nu} \operatorname{Re} D^{\mu \nu}(\omega, q) \operatorname{Im} V_{A, \|}^{\nu \mu}(\omega, q) .
$$

In the spinless model, the kernel $\mathcal{K}(\omega)$ is determined by processes described by the Feynman diagrams in Fig. 19 at $\gamma \ll \omega \ll \alpha T_{1}$ and Fig. 1b at $\alpha T_{1} \ll \omega \ll T_{1}$. In the spinful case, collisions between electrons of the same chirality but opposite spin (Fig. 1F), give the main contribution to $\mathcal{K}(\omega)$ for all $\omega \ll T_{1}$. The frequency dependence and the asymptotes of $\mathcal{K}(\omega)$ are shown in Fig. 2 ,

Large energy transfer, $\omega \gg T_{1}$. - In this case, we consider the contributions to $I^{\mu \nu}=I_{p}^{\mu \nu}+I_{s}^{\mu \nu}+I_{e h}^{\mu \nu}$ from the collective (plasmon/spinon) and e-h poles separately, see Figs. 1 1 -1f. Because of the splitting of the e-h, plasmon, and spinon poles, the collision integral would be nonlocal [11] if expressed solely in terms of the e-h distribution functions $N_{\omega}^{\mu \nu}$ [see Eq. (21)]. By introducing the plasmon/spinon distribution functions $n_{b}^{\mu}(\omega)$ we can express the emission rate of the bosonic excitations $(b=p, s)$ in the local form $\left(v_{p} \equiv u, v_{s} \equiv v_{F}\right)$ :

$$
I_{b}^{\mu \nu}(\omega)=\sum_{\alpha} \omega \mathcal{L}_{\alpha, b}^{\mu \nu}(\omega)\left[1+n_{b}^{\alpha}(\omega)\right]
$$

where the collision kernels $\mathcal{L}$ have the scaling form $\mathcal{L}_{\alpha, b}^{\mu \nu}=$ $\left(\gamma / 2 \omega^{2}\right)\left(v_{F}^{*} / v_{b}\right) \mathcal{A}_{\alpha, c}^{\mu \nu}$ and the $\mathcal{A}$-factors read

$$
\begin{aligned}
& \mathcal{A}_{ \pm \mu, p}^{\mu \mu}=\left[\eta\left(v_{F}^{*}-v_{F}\right)+v_{F} \pm u\right] / \eta v_{F}^{*}, \\
& \mathcal{A}_{ \pm, p}^{\mu,-\mu}=-\left(v_{F}^{*}-v_{F}\right) / v_{F}^{*}, \quad \mathcal{A}_{\alpha, s}^{\mu \nu}=(\eta-1) \delta_{\mu \nu} \delta_{\mu \alpha} .
\end{aligned}
$$

In turn, $n_{b}^{\mu}(\omega)$ satisfies the kinetic equation

$$
\left(\partial_{t}+\mu v_{b} \partial_{x}\right) n_{b}^{\mu}(\omega)=-\gamma_{b} n_{b}^{\mu}(\omega)+\frac{\eta \gamma}{2} \sum_{\alpha \beta} \mathcal{A}_{\mu, b}^{\alpha \beta} N_{\omega}^{\alpha \beta},
$$

where $\gamma_{b}=\gamma\left(v_{F} / v_{F}^{*}\right)$ and we used the relation $D_{K}^{\mu \nu} \simeq$ $2 \operatorname{Re} D_{R}^{\mu \nu}\left(1+2 N_{\omega}^{\mu \nu}\right)$. This kinetic equation describes the decay/creation of plasmons and spinons in/from $e$ $h$ pairs. Finally, the e-h pole in the emission rate (6) gives $I_{e h}^{\mu \nu}(\omega)=\delta_{\mu \nu}(\gamma / \omega) N_{\omega}^{\mu \mu}$. Note that the collision kernels $\mathcal{L}_{+, b}^{++} \simeq \mathcal{L}_{e h}=\gamma / \omega^{2}$ do not contain $\alpha$ as a small parameter. These most efficient relaxation processes involve electrons and bosons within the same chiral branch: their rates are resonantly enhanced since $v_{b}$ and $v_{F}^{*}$ are close to each other. As a result, $\alpha$ appears in the combination $\alpha^{2} /\left|v_{F}^{*}-v_{b}\right|^{\eta} \sim 1$. It can also be shown that the total kernel, $\mathcal{L}=\mathcal{L}_{e h}+(1 / 2) \sum_{\mu \nu \alpha, b} \mathcal{L}_{\alpha, b}^{\mu \nu}$, is equal to the asymptotic value of $\mathcal{K}(\omega)$ [Eq. (7)] in the limit $\omega \gg T_{1}$.

Energy relaxation rate.-We now turn to the energyrelaxation rate $\gamma_{E}$ in the limit of weak nonequilibrium.

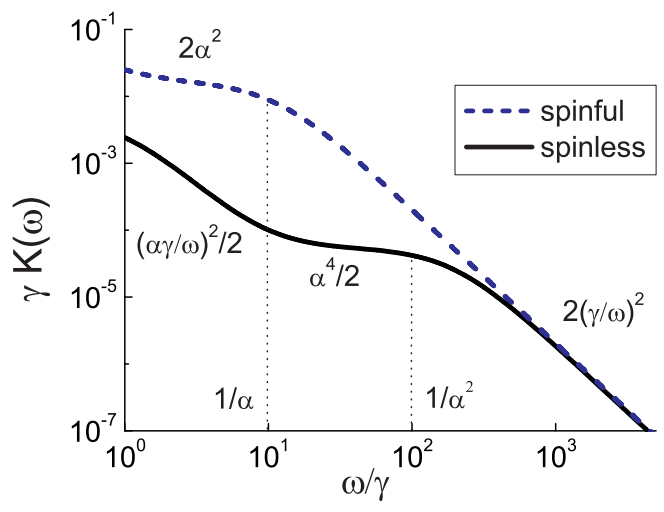

FIG. 2: Frequency dependence of the collision kernels for the spinless and spinful models [Eq. (7)] for $\alpha=0.1$.

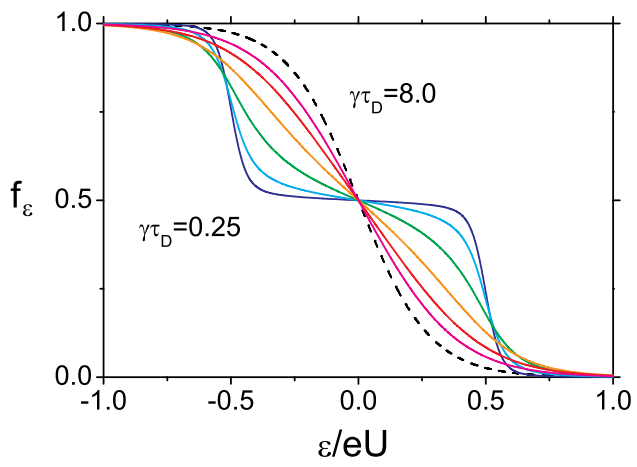

FIG. 3: Electron distribution function in the middle of a quantum wire of length $L$ biased by $e U=40 T$, for $T=T_{1} / 2$. The solid lines correspond to $\gamma \tau_{D}=0.25,0.5,1,2$, 4, and 8 , where $\tau_{D}=L / v_{F}^{*}$ and $\gamma$ is the elastic backscattering rate. The dashed curve shows the limiting Fermi distribution.

Linearizing the electron and boson kinetic equations, we estimate $\gamma_{E}$ from the total [20] collision kernel (Fig. 2)

$$
\gamma_{E} \sim T^{-1} \int_{0}^{T} d \omega \omega^{2} \mathcal{K}(\omega) \sim T^{2} \mathcal{K}(T)
$$

The characteristic $\omega$ for $\gamma_{E}$ is of order $T$. Since $\mathcal{K}(\omega) \simeq$ $2 \gamma / \omega^{2}$ for $\omega \gg T_{1}, \gamma_{E}$ at $T \gg T_{1}$ does not depend on $\alpha$ and is given by the backscattering rate:

$$
\gamma_{E} \sim \gamma, \quad T \gg T_{1}
$$

( $\alpha$ enters only through the condition on $T$, so that the result is also valid for the long-range Coulomb interaction). At $T \lesssim T_{1}$, Eq. (12) is only valid for short wires of length $L \lesssim v_{F} / \gamma$ - otherwise the system is localizedand shows that a full equilibration then has no time to develop. Note that $\gamma_{E}$ for spinful and spinless electrons turn out to be parametrically the same, in contrast to the weak-localization phase-relaxation rate $\gamma_{\phi}[18]$.

In the limit of strong nonequilibrium, we solve Eqs. (3), (10) numerically to obtain the distribution function of electrons $f_{\epsilon}$ in a wire biased by a voltage $U \gg T / e$, where $T$ is the temperature in the leads, as a function of 
the distance to the contacts $\left(f_{\epsilon}\right.$ shows up directly in tunneling spectroscopy [4]; for experiments on multi-channel wires, see, e.g., Ref. [21]). The result, shown in Fig. [3. confirms the estimate (12): the scale of $\tau_{D}=L / v_{F}^{*}$ on which $f_{\epsilon}$ equilibrates is $\gamma^{-1}$, despite $\alpha \ll 1$. For small $\alpha$, the inelastic processes involving opposite chiralites $\left(\mathcal{L}_{-}^{++}\right.$ and $\mathcal{L}_{+}^{+-}$), as well as the backscattering of plasmons on the boundaries [22, 23, 24, 25], can be neglected: the curves in Fig. 3 are thus $\alpha$-independent. At $\tau_{D} \gg \gamma^{-1}$, $f_{\epsilon}$ approaches the Fermi distribution with the temperature $T_{e}=\sqrt{3} e U / 4 \pi$.

Discussion.-As follows from Eqs. (12) and (10), the thermalization of electrons occurs on the same time scale as the lifetime of bosons. This elucidates a conceptually important point: boson decay is a source of the inelastic relaxation of electrons. Indeed, in the homogeneous case, the combination $\operatorname{Re} D \operatorname{Im} V$ in Eq. (7) yields zero energy transfer in the e-e scattering [5]. However, any plasmon scattering broadens the peak in $\operatorname{Im} V$, thus allowing for a finite transfer (even if $D$ remains free - no electron backscattering) 24]. This is true, in particular, in an inhomogeneous LL without impurities but with a nonuniform interaction $\alpha(x)$ 22, 23, 24, 25].

In a disordered LL, the spectral function of dRPA bosons is characterized by the rate $\gamma_{b} \simeq \gamma$ due to the elastic scattering off impurities [5]. One sees from Eq. (10) that out of equilibrium [when $n^{\mu}(\omega) \neq N_{\omega}^{\mu \mu}$ ] the boson scattering is represented entirely as the creation/annihilation of e-h pairs. As a result, the inverse process - the inelastic electron scattering due to the emission/absorption of bosonic excitations - is characterized by the same rate, $\gamma_{E} \sim \gamma$. Impurities induce also the anharmonic decay of plasmons, as well as their inelastic scattering on each other. These processes have been neglected as higher-loop corrections to the dRPA: their rates are much smaller than $\gamma_{E}$. It is the latter that gives the thermalization rate for the full bosonic distribution function $n(\omega)$ in Eq. (10) due to the coupling (through $\left.N_{\omega}\right)$ to the fermionic function $f_{\epsilon}$.

Conclusion.-We have formulated the analytical framework for disordered LLs out of equilibrium, based on the kinetic equations for the boson and electron distribution functions. We have found the equilibration rate, which, remarkably, coincides with the elastic scattering rate. The kinetic approach developed here is particularly convenient for studying heat transport and current noise in strongly-correlated disordered 1D systems [26].

We thank A. Altland, N. Birge, D. Gutman, A. Kamenev, M. Kiselev, J. Meyer, A. Mirlin, Y. Nazarov, H. Pothier, A. Yashenkin, and I. Yurkevich for interesting discussions. The work was supported by EUROHORCS/ESF (Project "Quantum Transport in Nanostructures"), by GIF Grant No. 965, and by CFN/DFG.
[1] T. Giamarchi, Quantum Physics in One Dimension (Oxford University Press, Oxford, 2004).

[2] H.T. Man and A.F. Morpurgo, Phys. Rev. Lett. 95, 026801 (2005); M.S. Purewal et al., ibid. 98, 186808 (2007), and references therein.

[3] O.M. Auslaender et al., Science 308, 88 (2005); W.K. Hew et al., Phys. Rev. Lett. 101, 036801 (2008), and references therein.

[4] Y.-F. Chen et al., Phys. Rev. Lett. 102, 036804 (2009).

[5] I.V. Gornyi, A.D. Mirlin, and D.G. Polyakov, Phys. Rev. Lett. 95, 046404 (2005); Phys. Rev. B 75, 085421 (2007).

[6] A. Furusaki and N. Nagaosa, Phys. Rev. B 47, 4631 (1993); P. Fendley, A.W.W. Ludwig, and H. Saleur, Phys. Rev. B 52, 8934 (1995); U. Weiss, Solid State Commun. 100, 281 (1996); R. Egger and H. Grabert, Phys. Rev. Lett. 77, 538 (1996); Phys. Rev. B 58, 10761 (1998).

[7] J.U. Kim, I.V. Krive, and J.M. Kinaret, Phys. Rev. Lett. 90, 176401 (2003).

[8] D.A. Bagrets et al., Semiconductors 42, 994, (2008).

[9] D.B. Gutman, Y. Gefen, and A.D. Mirlin, Phys. Rev. Lett. 101, 126802 (2008).

[10] I.V. Lerner and I.V. Yurkevich, in Nanophysics: Coherence and Transport, edited by H. Bouchiat et al. (Elsevier, Amsterdam, 2005).

[11] G. Catelani and I.L. Aleiner, JETP 100, 331 (2005).

[12] In contrast to Ref. [11], and also Refs. [8, 9], we construct our theory without the so-called "ghost modes".

[13] J. Rammer and H. Smith, Rev. Mod. Phys. 58, 323 (1986); A. Kamenev, in Nanophysics: Coherence and Transport, edited by H. Bouchiat et al. (Elsevier, Amsterdam, 2005).

[14] B.A. Muzykantskii and D.E. Khmelnitskii, JETP Lett. 62, 76 (1995).

[15] A. Kamenev and A. Andreev, Phys. Rev. B 60, 2218 (1999).

[16] T. Micklitz, A. Altland, and J. Meyer, arXiv:0805.3677,

[17] G. Zala, B.N. Narozhny, and I.L. Aleiner, Phys. Rev. B 64, 214204 (2001).

[18] A.G. Yashenkin et al., Phys. Rev. B 78, 205407 (2008).

[19] The scattering rate $\gamma$ includes the LL renormalization [1] due to virtual (elastic) processes on energy scales between $\max \{T, e U\}$ and the ultraviolet cutoff.

[20] A complementary approach has been formulated for spinless electrons in Ref. [8] in terms of "undressed" fermions, which gives the same result for $\mathcal{K}(\omega)$.

[21] H. Pothier et al., Phys. Rev. Lett. 79, 3490 (1997).

[22] Y. Oreg and A.M. Finkel'stein, Phys. Rev. Lett. 74, 3668 (1995).

[23] R. Fazio, F.W.J. Hekking, and D.E. Khmelnitskii, Phys. Rev. Lett. 80, 5611 (1998); I.V. Krive, J. Low Temp. Phys. 24, 377 (1998).

[24] In a finite-length LL with, e.g., $\alpha(x) \propto \theta(x) \theta(L-x)$, plasmons are backscattered off the noninteracting contacts. Inhomogeneities in the interaction strength induce the inelastic electron scattering [22] as well. This type of inelastic relaxation follows directly from the kinetic equations (3), (10) with the kernels (6), (17) taken for the space-dependent $\alpha(x)$. In a different framework-by directly calculating the Keldysh Green's function-the relaxation of the electron distribution in a finite LL has also been found in Ref. 25]. This mechanism of evolution of $f_{\epsilon}$ combines with the impurity-induced equilibration. 
[25] D.B. Gutman, Y. Gefen, and A.D. Mirlin, arXiv:0903.3333
[26] D.A. Bagrets, I.V. Gornyi, and D.G. Polyakov, in preparation. 